\title{
STELLAR POPULATIONS IN DWARF ELLIPTICAL GALAXIES
}

\author{
J. GORGAS, S. PEDRAZ AND N. CARDIEL \\ Dpto. de Astrofísica, Universidad Complutense, Madrid
}

AND

\section{J.J. GONZALEZ}

Instituto de Astronomía, U.N.A.M., México

We present the results of a spectroscopic study in which we have measured line-strength indices in a sample of 5 dwarf and 2 compact elliptical galaxies (mostly from Virgo). Some conclusions about the stellar populations in dwarf E's are derived by comparing the sample with classical $\mathrm{E}$ galaxies. In the adjacent figure, we plot in the $\mathrm{H} \beta-[\mathrm{MgFe}]$ plane the central indices of our sample of dwarf and compact E's together with data from González (1993, PhD Thesis). The grid represents the predictions of single-burst stellar population models from Worthey (1994, ApJS, 95, 107). The main conclusion is that, whilst the central regions of giant, intermediate and compact ellipticals span, according to stellar population models, a wide range in mean stellar ages, bright dwarf ellipticals are found to be old, compatible with a $10 \mathrm{Gyr}$ old stellar population. This means that the dichotomy found in the Fundamental Plane between dwarf ellipticals and the gE-iE-cE sequence (Bender, Burstein \& Faber 1992, ApJ, 399, 462) is also observed in the stellar populations.

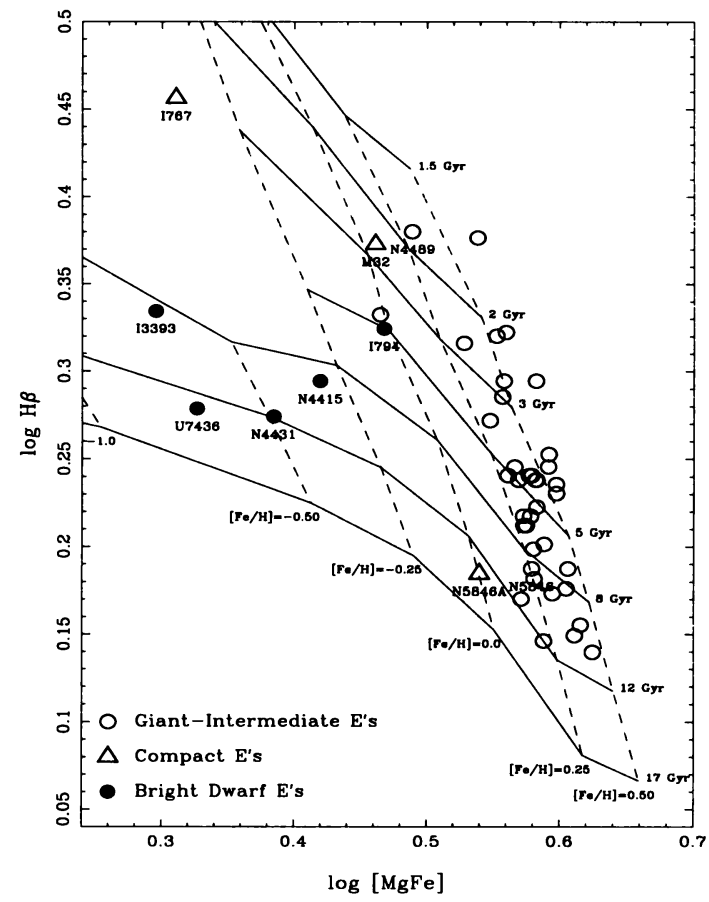

